\title{
Differential regulation of the biosynthesis of glucose transporters by the PI3-K and MAPK pathways of insulin signaling by treatment with novel compounds from Liriope platyphylla
}

\author{
YOEN KYUNG LEE ${ }^{1 *}$, JI EUN KIM ${ }^{1 *}$, SO HEE NAM ${ }^{1}$, JUN SEO GOO ${ }^{1}$, SUN IL CHOI ${ }^{1}$, YOUNG HWAN CHOI ${ }^{1}$, \\ CHANG JUN BAE ${ }^{2}$, JONG MIN WOO ${ }^{2}$, JUNG SIK $\mathrm{CHO}^{3}$ and DAE YOUN HWANG ${ }^{1}$ \\ ${ }^{1}$ College of Natural Resources and Life Science, Pusan National University, Miryang 627-706; ${ }^{2}$ Department of \\ Laboratory Animal Resources, National Institute of Food and Drug Evaluation, Korea FDA, Seoul 122-704; \\ ${ }^{3}$ Multidisciplinary Technology Institute, Hoseo University, Asan 336-795, Republic of Korea
}

Received September 15, 2010; Accepted November 12, 2010

DOI: $10.3892 / \mathrm{ijmm} .2010 .581$

\begin{abstract}
The insulin signaling pathway, involving protein kinase $\mathrm{B}(\mathrm{PKB})$ and mitogen-activated protein kinase (MAPK), mediates the biological response to insulin and several growth factors and cytokines. To investigate the correlation between glucose transporter (Glut) biosynthesis and the insulin signaling pathway activated by novel compounds of Liriope platyphylla (LP9M80-H), alterations in Glut and key protein expression in the insulin signaling pathway were analyzed in the liver and brain of ICR mice treated with LP9M80-H. An in vitro assay showed that the highest level of insulin concentration was observed in the LP9M80-H-treated group, followed by the LP-H, LP-M, LP-E, and LP9M80-C-treated groups. Therefore, LP9M80-H was selected for use in studying the detailed mechanism of the insulin signaling pathway in animal systems. In an in vivo experiment, LP9M80-H induced a significant increase in glucose levels and a decrease of insulin concentration in the blood of mice, while their body weight remained constant over 5 days. The expression level of Glut-3 was down-regulated in the liver, or maintained at the same level in the brain of LP9MH80-H-treated mice. These changes corresponded to the phosphorylation of the p38 protein rather than to ERK and JNK in the MAPK signaling pathway. In addition, the expression level of Glut-1 increased significantly after LP9MH80-H treatment of both insulin target tissues in mice. Western blot analysis showed that Akt in the PI3-K pathway
\end{abstract}

Correspondence to: Dr Dae Youn Hwang, Department of Biomaterial Science, College of Natural Resources and Life Science, Pusan National University, 50 Cheonghak-ri, Samnangjin-eup Miryang-si, Gyeongsangnam-do 627-706, Republic of Korea E-mail: dyhwang@pusan.ac.kr

${ }^{*}$ Contributed equally

Key words: insulin signaling pathway, phosphatidylinositol-3kinase, mitogen-activated protein kinase, glucose transporter, Liriope platyphylla mainly participated in Glut-1 biosynthesis. Thus, these results suggest the possibility that the LP9M80-H-induced regulation of Glut- 1 and Glut-3 biosynthesis may be mediated by the Akt and p38 MAPK signaling of the insulin signaling pathway in the liver and brain of mice.

\section{Introduction}

The insulin signaling pathway in several target tissues transfers signals that promote normal growth and development and the homeostasis of glucose, fat, and protein metabolism into the cell through the stimulation of glucose uptake, glycogen synthesis, protein translation, and synthesis $(1,2)$. This pathway generally includes two major downstream pathways: the Raf/Ras/mitogen-activated protein kinase (MAPK) and the phosphatidylinositol-3-kinase (PI3-K) pathways. In the initiation stage of the insulin signaling pathway, the formation of the insulin-insulin receptor complex induced by insulin binding can transfer the signal into two downstream pathways via intermediate molecules such as Shc and the insulin receptor substrate (IRS) $(3,4)$. Of the two downstream pathways, the MAPK pathway is activated by the binding of Grb2 to Tyrphosphorylated Shc or IRS via its $\mathrm{SH} 2$ domain. This signal leads to the expression of genes associated with glucose metabolism and the induction of mitosis in cells (1). In particular, glucose transporter (Glut)-3 biosynthesis is mediated by the MAPK pathway to regulate glucose uptake (2). But the PI3-K pathway is primarily a mediator for the metabolic response to insulin, and leads to a large variety of biological actions, including protein and glycogen synthesis, glucose transportation, and anti-lipolytic and anti-apoptotic activities (1). In the PI3-K pathway, Akt (protein Ser/Thr kinase B) is activated by PDK1, and plays an important role in cell proliferation and insulin action in cells (1). Furthermore, Akt has been implicated in regulating the translocation of Glut-4, an insulin-sensitive glucose transporter expressed by muscle and fat cells, and the biosynthesis of Glut-1, a high-affinity glucose transporter expressed in most types of cells (5). Although the signaling mechanism via the PI3-K and MAPK pathways has been investigated under a variety of stimulations, the precise mechanism of action of the insulin-signaling 
pathway is not yet fully understood, particularly with respect to how it would respond to various novel compounds.

In oriental medicine, Liriope platyphylla has long been a well-known herb used in treatment of asthma and bronchial and lung inflammation (6). Liriope platyphylla are perennial seed-reproducing plants, and are widely distributed in the temperate climate region of the northern hemisphere. In Korea, these plants mainly grow in low mountain areas, less than $500 \mathrm{~m}$ above sea level, and their leaves remain green throughout the year (7). The effects of root extracts of Liriope platyphylla in preventing obesity, diabetes, and neurodegenerative diseases have recently been proven in several studies (8-11). Of these therapeutic effects, the effects of Liriope platyphylla against obesity and diabetes have long been well-known in Korea. Gyeongshingangjeehwan (GGEx), mainly containing Liriope platyphylla, may effectively prevent obesity and hypertriglyceridemia through appetite inhibition and activation of hepatic PPAR $\gamma$ in OLETF male rats (8). A homoisoflavone-enriched fraction in a methanol extract was also found to increase insulin-stimulated glucose uptake in 3T3-L1 adipocytes through increased Glut-4 content in plasma membranes (9). However, few studies have been conducted to investigate the mechanism of correlation between Glut biosynthesis and the insulin signaling pathway using novel extracts isolated from Liriope platyphylla towards assessing their use in diabetes therapy.

As demonstrated by our data herein, LP9M80-H, a novel extract isolated from Liriope platyphylla, induced significant changes in glucose metabolism in vitro and in vivo. Furthermore, our results show that Akt and p38 as members of the insulin signaling pathway may play an important role in the regulation of the LP9M80-H-induced alterations in Glut-1 and Glut-3 expression in glucose metabolism.

\section{Materials and methods}

Cell culture and treatment. The insulin producing HIT-T15 pancreatic B-cell line was purchased from the Korean Cell Line Bank (Seoul, Korea). This cell line was maintained for 24-36 h in RPMI-1640 (Invitrogen Corp., Carlsbad, CA, USA) containing $10 \%$ fetal bovine serum, $2 \mathrm{ml} \mathrm{L-glutamine,} 1 \%$ nonessential amino acids, $100 \mathrm{IU} / \mathrm{ml}$ penicillin, and $100 \mu \mathrm{g} / \mathrm{ml}$ of streptomycin. In order to detect an insulin concentration, the wells in 96-well plate were divided into 11 groups involving 1 vehicle and 10 novel extracts, and were further classified into 9 subgroups of different concentrations (vehicle group and the $500,250,125,62.4,31.3,15.6,7.8$ and $3.9 \mu \mathrm{g} / \mathrm{ml}$ groups). HIT-T15 cells were seeded at a density of $4 \times 10^{4}$ cells/200 $\mu 1$ in 96-well plates and grown for $24 \mathrm{~h}$ in a $37^{\circ} \mathrm{C}$ incubator. When the cells attained $70-80 \%$ confluence, various concentrations of novel extracts dissolved in DMSO were added to each well and further incubated for another $24 \mathrm{~h}$. After $24 \mathrm{~h}$, culture supernatants were collected from these wells to detect the insulin concentration.

Care and use of animals. The animal protocol used in this study has been reviewed by the Pusan National UniversityInstitutional Animal Care and Use Committee (PNU-IACUC) on ethical procedures and scientific care, and was approved (Approval No. PNU-2008-0056). Adult ICR mice were purchased from Samtacho Co. in Korea and handled at the Pusan National University Laboratory Animal Resources Center according to NIH guidelines. All mice were given a standard irradiated chow diet (Purina Mills, Inc., Seoungnam, Korea) ad libitum, and were maintained in a specified pathogen-free state under a strict light cycle (light on at $06: 00 \mathrm{~h}$ and off at 18:00 h) at a temperature of $22 \pm 2^{\circ} \mathrm{C}$ and at $50 \%$ relative humidity.

Experimental design and measurement of blood glucose concentration. Eight-week-old ICR mice $(n=14)$ were assigned to one of two groups: the vehicle group and the LP9M80-Htreated group. The first group of ICR mice received a constant volume of olive oil daily via gavage (vehicle-treated group), while the second group received $10 \mu \mathrm{g} / \mathrm{g}$ body weight/day of LP9M80-H (dissolved in olive oil in order to obtain a final concentration of $3 \mathrm{mg} / \mathrm{ml}$ ) via gavage (LP9M80-H-treated group). At 5 days after LP9M80-H treatment, all of the animals were immediately sacrificed using $\mathrm{CO}_{2}$ gas in order to prepare blood and tissue samples, which were stored in Eppendorf tubes at $-70^{\circ} \mathrm{C}$ until assayed. In addition, the blood glucose concentration of the mice was detected after $24 \mathrm{~h}$ of fasting using the sensitive strip of the blood glucose monitoring system (i-SENS Co., Seoul, Korea).

Western blot analysis. The proteins prepared from the tissues of the LP9M80-H-treated and vehicle-treated mice were separated by electrophoresis on a 4-20\% SDS-PAGE gel for $3 \mathrm{~h}$ and transferred to nitrocellulose membranes for $2 \mathrm{~h}$ at $40 \mathrm{~V}$. Each membrane was incubated separately with the following primary antiodies: anti-Glut-1 (ab40084, Abcam, Cambridge, UK), anti-Glut-3 (ab15311, Abcam), anti-ERK (sc-94, Santa Cruz Biotechnology Inc., Santa Cruz, CA, USA), anti-p-ERK (sc-7383, Santa Cruz Biotechnology Inc.), anti-JNK (\#9252, Cell Signaling Technology, Boston, MA, USA), anti-p-JNK (\#9251, Cell Signaling Technology), anti-p38 (\#9212, Cell Signaling Technology), anti-p-p38 (\#9211, Cell Signaling Technology), anti-Akt (\#9272, Cell Signaling Technology), anti-p-Akt (\#4058, Cell Signaling Technology) and anti-actin (A5316, Sigma-Aldrich, St. Louis, MO, USA) antibodies overnight at $4{ }^{\circ} \mathrm{C}$. The membranes were washed with a washing buffer $\left(137 \mathrm{mM} \mathrm{NaCl}, 2.7 \mathrm{mM} \mathrm{KCl}, 10 \mathrm{mM} \mathrm{Na} \mathrm{HPO}_{4}, 2 \mathrm{mM}\right.$ $\mathrm{KH}_{2} \mathrm{PO}_{4}$, and $0.05 \%$ Tween-20) and incubated with a horseradish peroxidase (HRP)-conjugated goat anti-rabbit IgG secondary antibody (Zymed Laboratories Inc., South San Francisco, CA, USA) at a 1:1,000 dilution at room temperature for $2 \mathrm{~h}$. The membrane blots were developed using a Chemiluminescence Reagent Plus kit (ECL, Pfizer and Pharmacia, NY, USA).

Quantification of insulin by ELISA. The insulin concentration in serum samples collected from the LP9M80-H-treated and vehicle-treated mice was determined using the ultra-sensitive assay procedure and the reagents included in the Mercodia Mice Insulin ELISA kit (no. 10-1137-01, Mercodia, Uppsala, Sweden). The serum and the standards were incubated in antibody-coated plates for $2 \mathrm{~h}$ at room temperature on a plate shaker at 500-600 rpm. The wells were then washed 6 times using an automatic plate washer (PV100; Hoefer Inc., Holliston, MA, USA). The HRP conjugate was added to all 

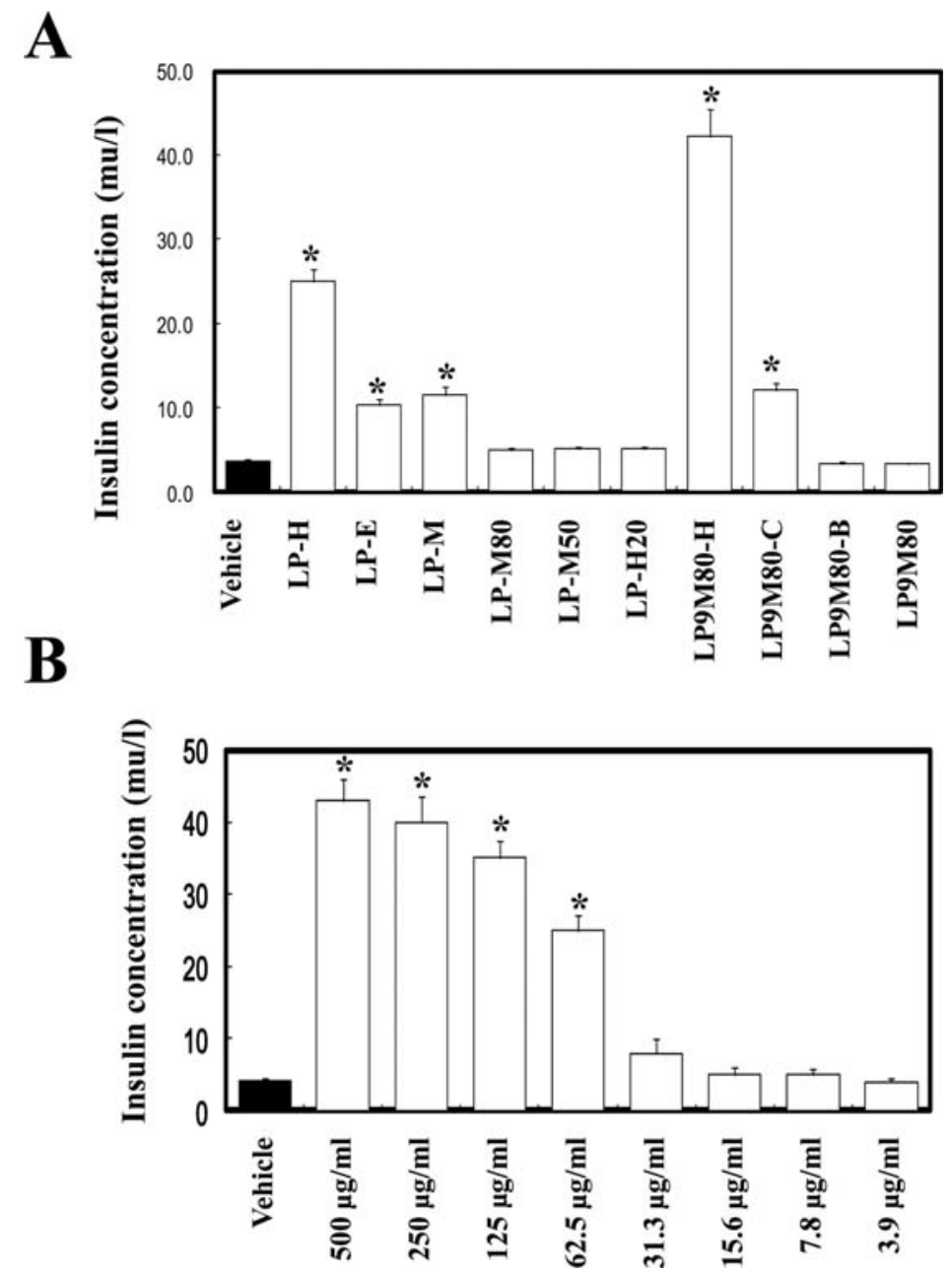

Figure 1. Stimulatory effects of extracts on the insulin secretion of HIT-T15 cells. (A) Cells were cultured with one of the ten extracts in DMSO at $500 \mu$ g/ml concentrations for $24 \mathrm{~h}$. (B) Cells were cultured with LP9M80-H extracts in DMSO at various concentrations for $24 \mathrm{~h}$. The culture supernatants were collected from each cell. An insulin concentration in the supernatant was measured using an anti-insulin ELISA kit. The values of the data represent the mean \pm SD of three experiments. ${ }^{*} \mathrm{p}<0.05$ is the significance level compared to the vehicle-treated group.

plates and incubated for $30 \mathrm{~min}$ at room temperature on a shaker. The reaction was terminated with the addition of a $50 \mu 1$ stop solution. The plates were read at $450 \mathrm{~nm}$ using a Molecular Devices VMax plate reader (Molecular Device Co., Sunnyvale, CA, USA).

Immunohistochemistry. Immunohistochemical analysis was performed as described in a previous study (12). Briefly, the distribution of insulin protein was observed using optical microscopy after fixing the tissue samples in 5\% formalin for $12 \mathrm{~h}$, embedding them in paraffin, and slicing them into $4-\mu \mathrm{m}$ thick sections. These sections were de-paraffinized with xylene, rehydrated, and pre-treated for $30 \mathrm{~min}$ at room temperature with a PBS-blocking buffer containing $10 \%$ goat serum. The samples were then incubated with the mouse anti-insulin antibody diluted 1:1,000 on a PBS-blocking buffer. These antigen-antibody complexes were visualized with the biotinylated goat anti-rabbit-conjugated HRP streptavidin secondary antibody (Histostain-Plus Kit, Zymed Laboratories Inc.) diluted 1:1,500 in a PBS-blocking buffer. A stable DAB (Invitrogen Corp.) and an Imazing Densitometer (GS-690; Bio-Rad, Hercules, CA, USA) were used to detect insulin proteins.
Statistical analysis. One-way ANOVA (SPSS for Windows, Release 10.10, Standard Version, Chicago, IL, USA) was used to determine whether significant differences existed between the LP9MH80-H-treated and vehicle-treated groups. All values were reported as the mean \pm SD. A p-value of $<0.05$ was considered significant.

\section{Results}

Screening of 10 novel compounds for their insulin secretion ability in an insulinoma cell line. In order to screen the insulin secretion ability of ten novel extracts purified from Liriope platyphylla, insulin concentrations were detected in the culture supernatant using the previously described insulin ELISA kit. The highest level of insulin concentration was observed in the LP9M80-H-treated group, followed by the LP-H-, LP-M-, LP-E-, and LP9M80-C-treated groups, while other extracts did not induce insulin secretion from HIT-T15 cells (Fig 1A). Therefore, one of these extracts, the LP9M80-H was selected as the best candidate for a therapeutic drug. Also, in order to determine the optimum LP9M80-H concentration for insulin secretion, insulin concentrations were detected in HIT-T15 cells-treated with various concentrations of 


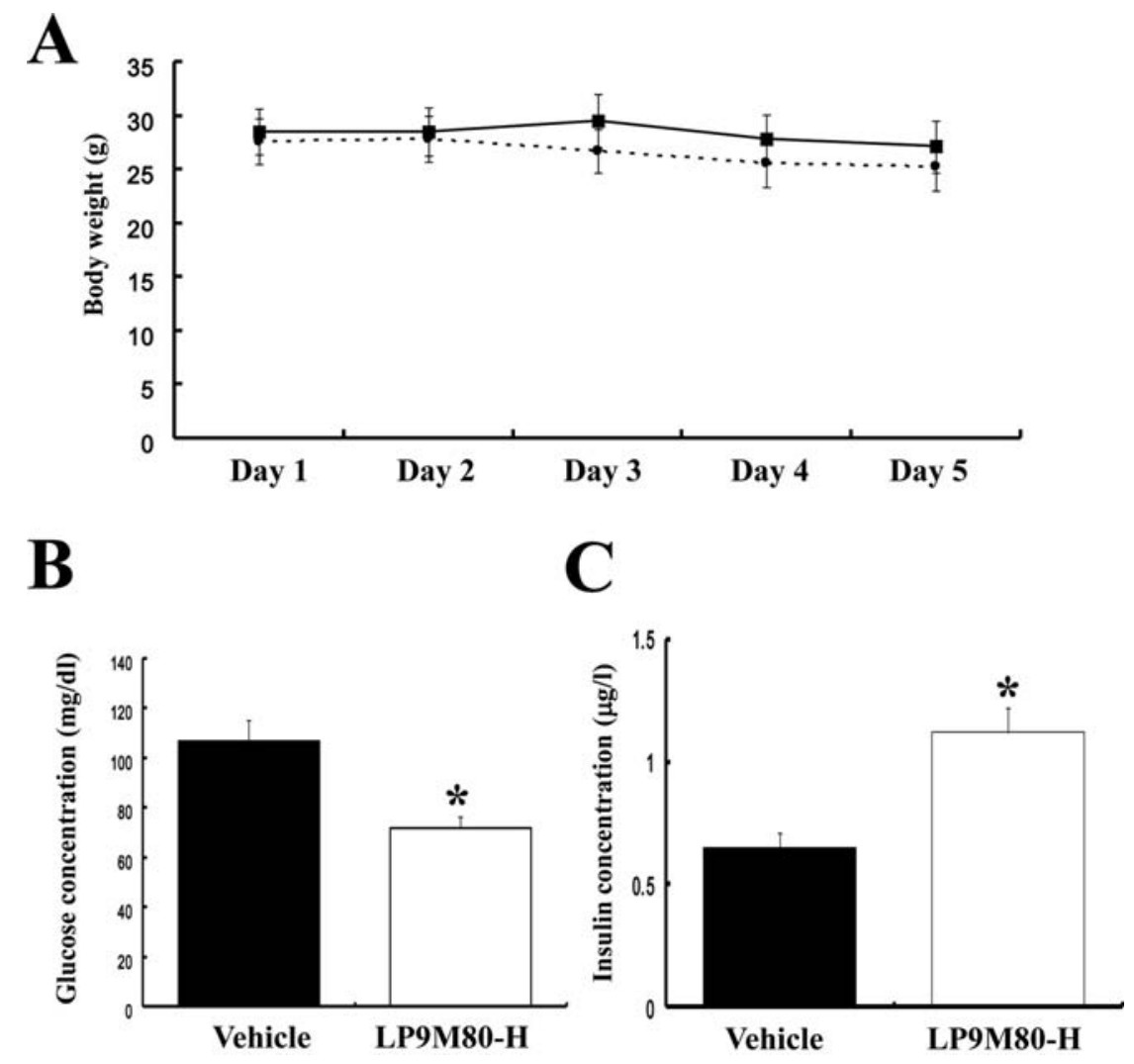

Figure 2. Effects of LP9M80-H treatment on body weight (A), glucose concentration (B) and insulin concentration (C) of ICR mice. Mice were treated by oral gavage with LP9M80-H (10 $\mu \mathrm{g} / \mathrm{g}$ body weight/day) for 5 days. The control group received vehicle only. Blood glucose and insulin concentration were detected as described in Materials and methods. Values are means $\pm \mathrm{SD}$, and the coefficient of variation of glucose and insulin in our serum samples were $5.2 \%$ and $2.4 \%$, respectively. ${ }^{*} \mathrm{p}<0.05$ is the significance level compared to the vehicle-treated group.

LP9M80-H. As shown in Fig. 1B, high levels of insulin were maintained during treatment with $62.5 \mu \mathrm{g} / \mathrm{ml} \mathrm{LP} 9 \mathrm{M} 80-\mathrm{H}$, but these levels decreased in cells-treated with 31.3 to $3.9 \mu \mathrm{g} / \mathrm{ml}$ LP9M80-H. Therefore, these results suggest that the optimum LP9M80-H concentration for the effective treatment was in the range of $100-120 \mu \mathrm{g} / \mathrm{ml}$. These concentrations were applied to animal administration tests to induce insulin secretion in pancreatic $\beta$-cells.

Effect of LP9M80-H on insulin secretion and glucose homeostasis of ICR mice. Jeong et al (8) suggested that Liriope platyphylla may be considered an effective therapeutic drug for diabetes-related diseases using OLETF rats. To investigate whether the LP9M80-H treatment would affect the regulation of glucose metabolism, we measured the serum glucose and insulin concentrations in both the LP9M80-H-treated and vehicle-treated groups after 5 days of treatment. There was no difference in body weight between the LP9M80-H- and the vehicle-treated groups (Fig. 2A). The serum glucose concentration was significantly decreased in the LP9M80-Htreated group as compared to the vehicle group (Fig. 2B). Furthermore, insulin concentrations showed a pattern opposite to that of serum glucose concentrations. LP9M80-H-treated mice showed a significant increase in insulin concentration, compared to the $0.65 \mu \mathrm{g} / \mathrm{l}$ insulin level observed in vehicletreated mice (Fig. 2C).

In order to detect the localization and distribution of the insulin protein in pancreatic tissues, insulin protein immuno- reactivity in the pancreatic islet was analyzed using optical microscopy. The insulin immunostaining intensity in the LP9M80-H-treated mice was spread throughout the B-cells of the pancreatic islets. However, the level of intensity in the vehicle-treated mice was slightly lower than that of the LP9M80-H-treated mice (Fig. 3). These results suggest that LP9M80-H treatment improves the glucose regulation process through stimulation of insulin secretion in ICR mice.

Effect of LP9M80-H on the regulation of Glut expression via the insulin signaling pathway in the liver of ICR mice. Glut biosynthesis can be regulated by the MAPK and PI3-K pathways of the insulin signaling pathway. In particular, the MAPK pathway mediates Glut-3 expression, while the PI3-K pathway regulates Glut-1 biosynthesis (2). Therefore, to investigate the regulation mechanism of Glut biosynthesis through the insulin signaling pathway after LP9M80-H treatment, the phosphorylation levels of MAPK and PKB members were detected in the livers of ICR mice treated with LP9M80-H. In the MAPK signaling pathway, the highest increase of phosphorylation was observed in ERK. The phosphorylation level of JNK increased significantly due to LP9M80-H treatment, whereas p38 phosphorylation decreased in the same conditions (Fig. 4). Furthermore, to investigate the effect of the MAPK signaling pathway on Glut-3 biosynthesis, the Glut-3 membrane content in liver tissue was measured. After LP9M80-H treatment, Glut-3 expression decreased significantly in liver tissue compared to vehicle- 

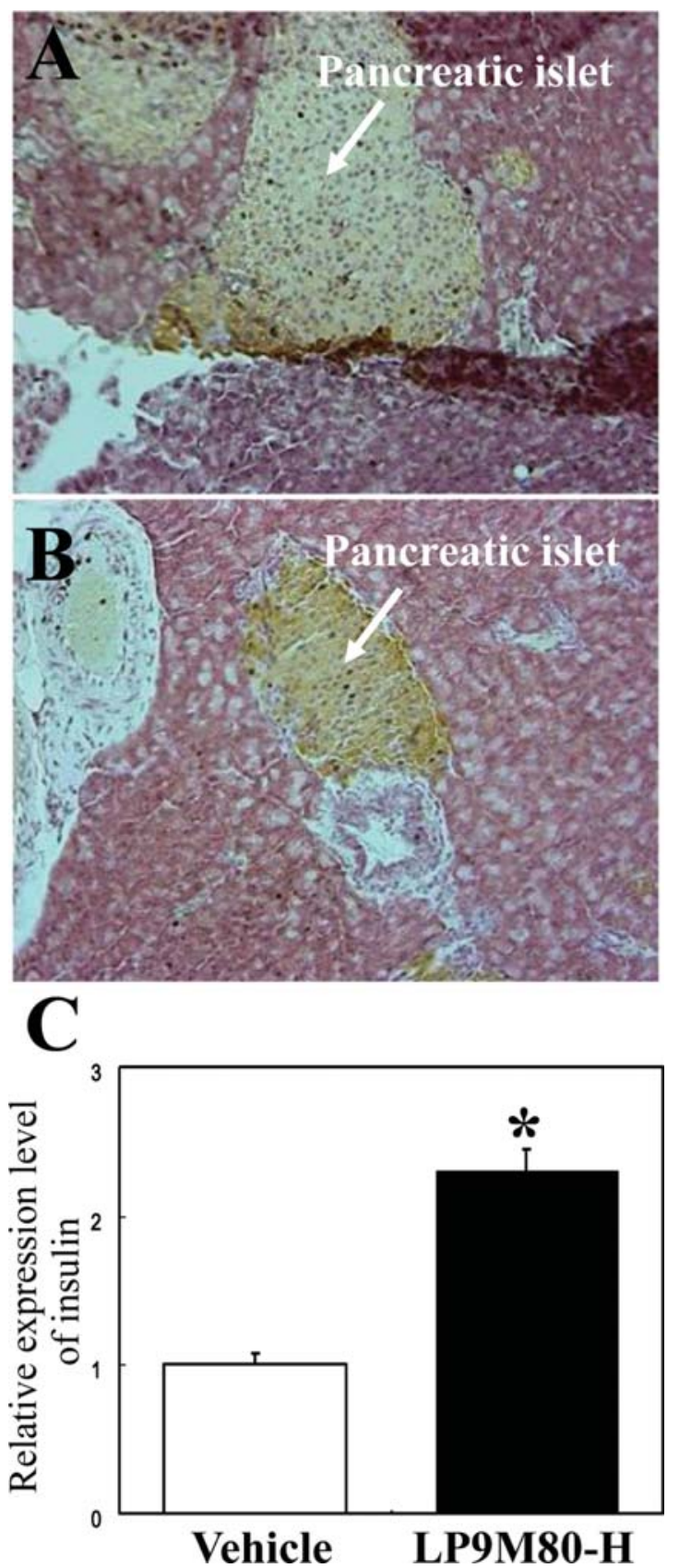

Figure 3. Immunostaining analysis of insulin protein in the pancreas of vehicle treated (A) and LP9M80-H treated mice (B). The expression profile of insulin in the pancreas was analyzed at 8 weeks of age by immunostaining analysis after LP9M80-H treatment for 5 days. At 200x magnification, highintensity staining was observed in the pancreas of LP9M80-H-treated mice compared with the vehicle-treated mice. The data represents the mean $\pm \mathrm{SD}$ from three replicates. ${ }^{*} \mathrm{p}<0.05$ is the significance level compared to the vehicle-treated group.

treated mice (Fig. 6). Taken together, these results show that changes in Glut-3 expression correspond to changes in $\mathrm{p} 38$ phosphorylation. Therefore, the p38 pathway in the three members of the MAPK signaling pathway may be a main contributor in the regulation of Glut-3 expression in the liver of ICR mice after LP9M80-H treatment.

Meanwhile, in the PI3-K signaling pathway, the Akt phosphorylation level was significantly increased in the LP9M80-H-treated group compared to the vehicle group

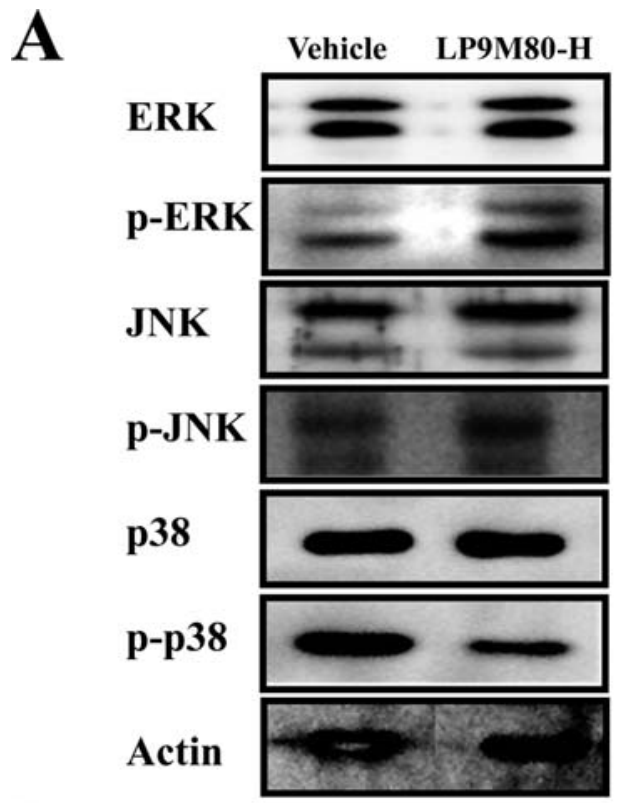

B

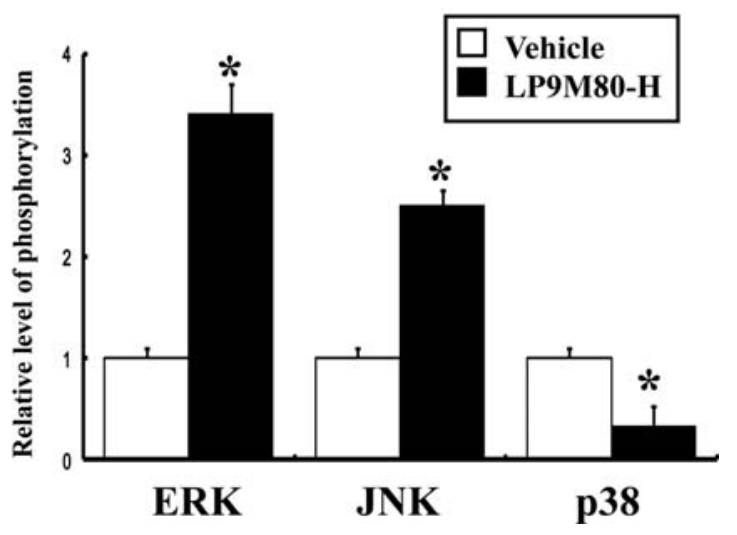

Figure 4. Phosphorylation levels of three members of the MAPK family in the livers of vehicle-treated and LP9M80-H-treated mice. To detect the expression level of each protein, the membranes were incubated with the protein-specific antibodies and the $\beta$-actin protein from the liver lysates. Three mice per group were assayed by Western blotting. The values are reported as the mean $\pm \mathrm{SD}$. ${ }^{*} \mathrm{p}<0.05$ is the significance level compared to the vehicle-treated group.

(Fig. 5). LP9M80-H treatment also induced up-regulation in Glut-1 expression (Fig. 6). Therefore, these results show that the increase in Glut-1 expression induced by LP9M80-H treatment can be mediated by Akt of the PI3-K pathway in mouse liver.

Effect of LP9M80-H on the regulation of Glut expression via the insulin signaling pathway in ICR mouse brain. A constant supply of glucose is essential to the brain to maintain proper function (13). To study whether LP9M80-H affects the insulin signaling pathway in maintaining glucose uptake, the alteration of two main pathways belonging to the insulin signaling pathway were analyzed in the brain tissue of LP9M80-H-treated and vehicle-treated mice using Western blot analysis. Of the three components of the MAPK pathway assessed, the level of p-ERK was dramatically increased in the LP9M80-H-treated group compared to the vehicle-treated 
A

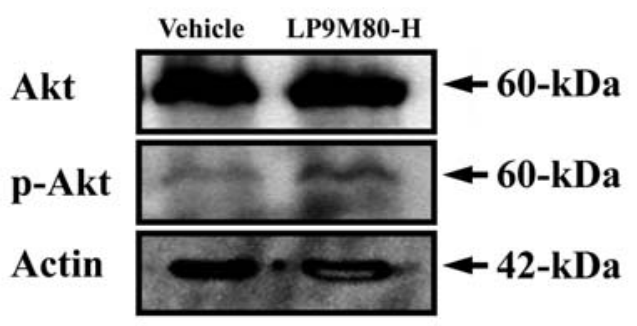

B

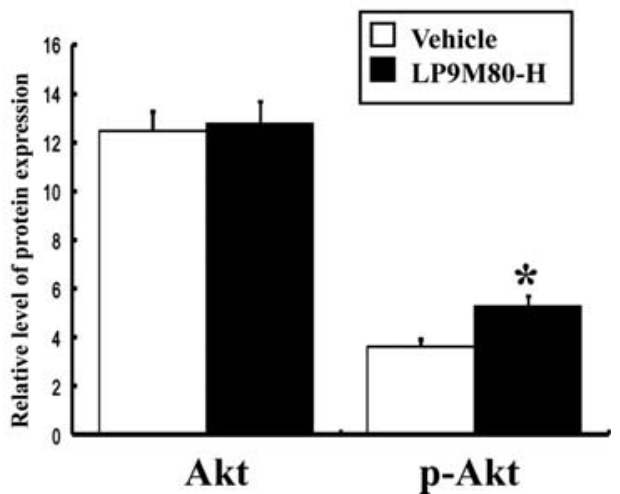

Figure 5. Phosphorylation levels of Akt in the liver of vehicle-treated and LP9M80-H-treated mice. Cell lysates were prepared from liver tissues of vehicle-treated and LP9M80-H-treated mice, as described in Materials and methods. Fifty micrograms of protein per sample were immunoblotted with antibodies for each protein. Three samples were assayed in triplicate using Western blotting. The values are the mean $\pm \mathrm{SD} .{ }^{*} \mathrm{p}<0.05$ is the significance level compared to the vehicle-treated group.

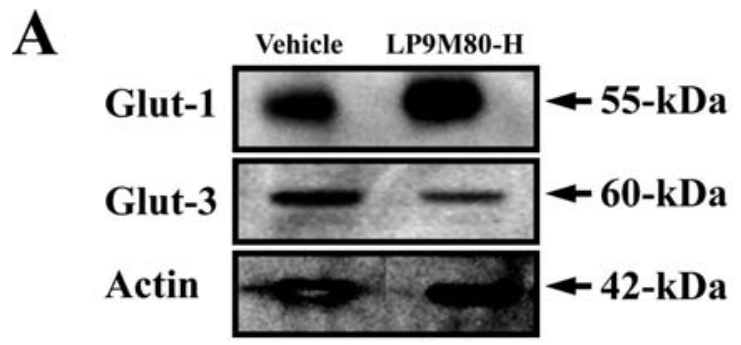

\section{B}

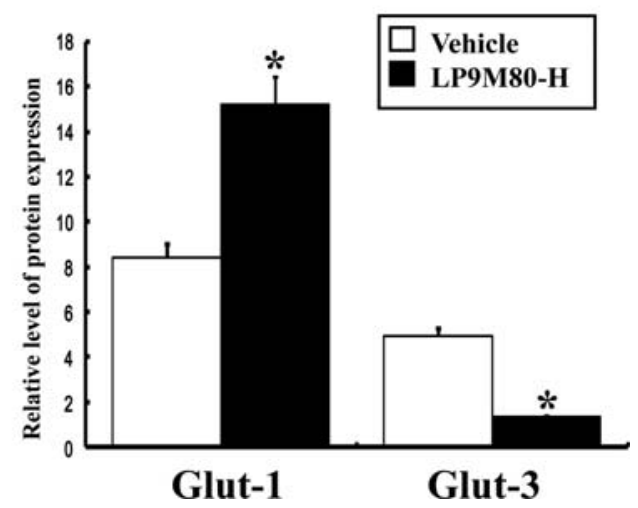

Figure 6. Expression levels of Glut-1 and Glut-3 in the livers of vehicletreated and LP9M80-H-treated mice. The Glut-1 and Glut-3 protein expression in the liver was detected with anti-Glut-1, anti-Glut-3 primary antibody, and horseradish peroxidase-conjugated goat anti-rabbit IgG, as described in Materials and methods. The intensity of the Glut protein was calculated using an imazing densitometer. The values are the mean $\pm \mathrm{SD}$. ${ }^{*} \mathrm{p}<0.05$ is the significance level compared to the vehicle-treated group.
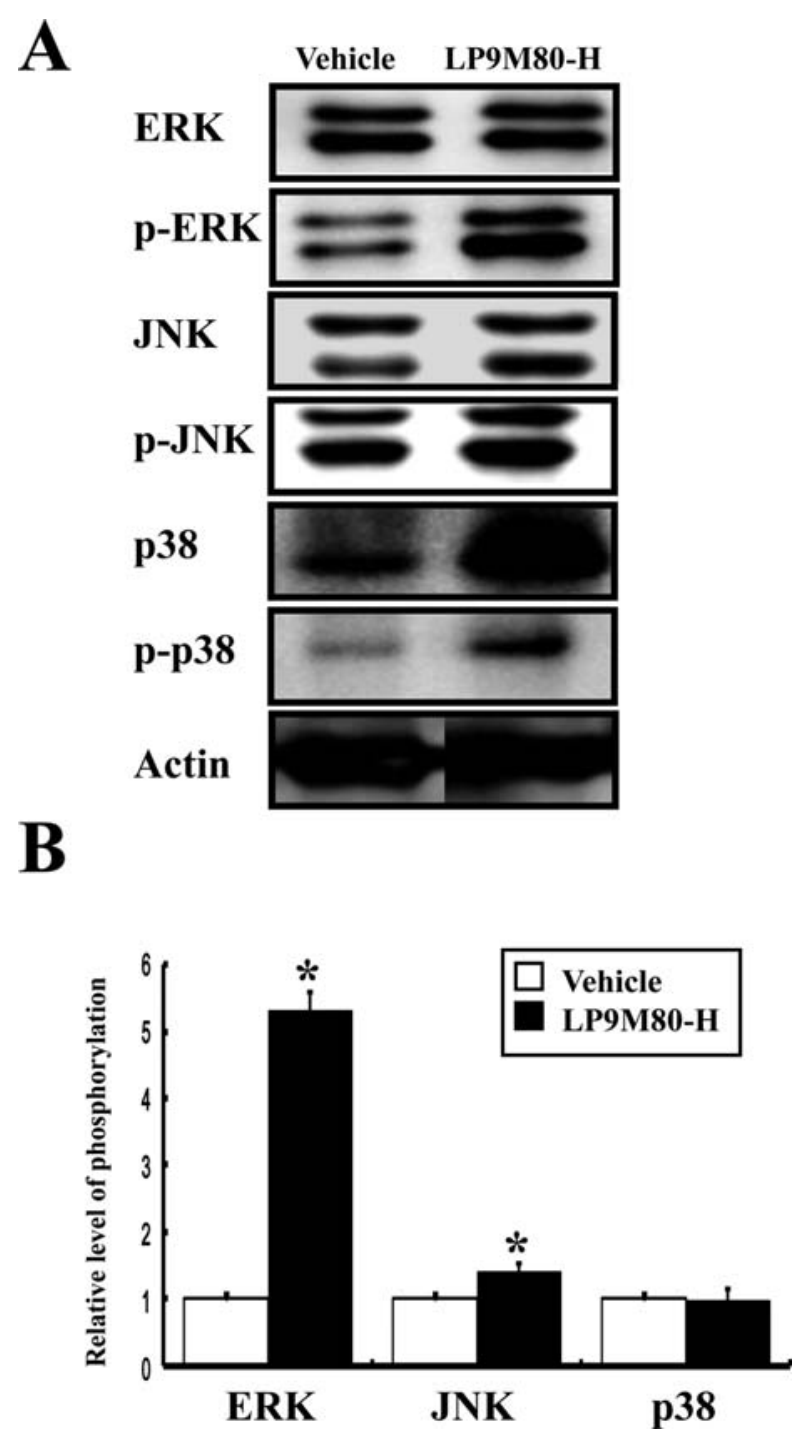

Figure 7. Phosphorylation levels of three members of the MAPK family in the brain of vehicle-treated and LP9M80-H-treated mice. To detect the expression level of each protein, the membranes were incubated with specific antibodies for each protein and the $\beta$-actin protein from the brain lysates. Three mice per group were assayed by Western blotting. The values are reported as the mean $\pm \mathrm{SD}$. ${ }^{*} \mathrm{p}<0.05$ is the significance level compared to the vehicle-treated group.

group, while JNK was slightly increased in that group. However, the phosphorylation level of p38 remained constant in the LP9M80-H-treated group (Fig. 7). Meanwhile, Glut-3 biosynthesis regulated by the MAPK signaling pathway did not change after LP9M80-H treatment (Fig. 9). Therefore, these results suggest that the p38 pathway may be a major factor in the regulation of Glut-3 biosynthesis in the brain of ICR mice treated with LP9M80-H.

In the PI3-K pathway, the phosphorylation level of Akt was higher in the LP9M80-H-treated group than in the vehicle-treated group (Fig. 8). The expression level of Glut-1 associated with the Akt pathway was significantly increased in the LP9M80-H-treated group (Fig. 9). These results show that the Akt pathway in the insulin signaling pathway may contribute to Glut-1 biosynthesis in LP9M80-H treatment conditions. 
A

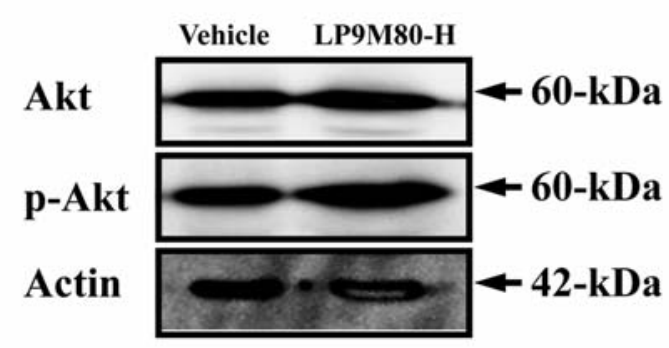

B

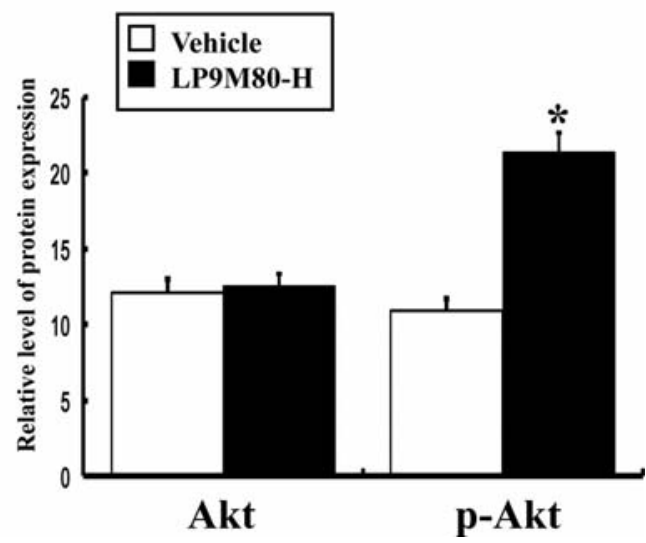

Figure 8. Phosphorylation levels of Akt in the brains of vehicle-treated and LP9M80-H-treated mice. Cell lysates were prepared from brain tissues of vehicle-treated and LP9M80-H-treated mice, as described in Materials and Methods. Protein, $50 \mu \mathrm{g}$ per sample, was immunoblotted with antibodies for each protein. Three samples were assayed in triplicate using Western blotting. The values are the mean \pm SD. ${ }^{*} \mathrm{p}<0.05$ is the significance level compared to the vehicle-treated group.

A

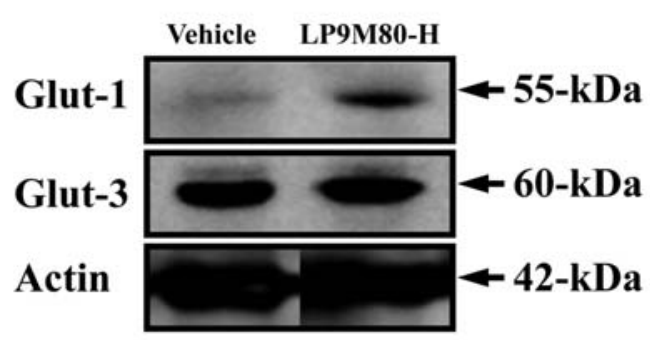

B

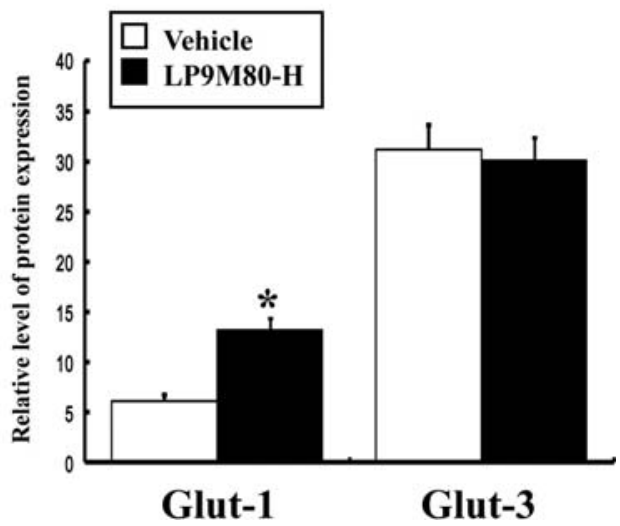

Figure 9. Expression levels of Glut-1 and Glut-3 in the brain of vehicletreated and LP9M80-H-treated mice. The Glut-1 and Glut-3 protein expression in the brain was detected with anti-Glut-1, anti-Glut-3 primary antibodies, and horseradish peroxidase-conjugated goat anti-rabbit IgG as described in Materials and methods. The intensity of the Glut protein was calculated using an imazing densitometer. The values are the mean \pm SD. ${ }^{*} \mathrm{p}<0.05$ is the significance level compared to the vehicle-treated group.

\section{Discussion}

Of the many medicinal properties of Liriope platyphylla, its anti-inflamation and anti-microbial effects have been studied the most. Kim et al (14) screened the effects of antibacterial drugs for the extracts of many plants via the detection of inhibitory activity of sortase. Among 80 medical plants tested, Liriope platyphylla, Cocculus trilobus, Fritillaria verticillata, and Rhus verniciflua had strong bacteriocidal activity. Also, in a murine model of asthma, Liriope platyphylla was shown to have strong inhibitory effects on airway inflammation and hyperresponsiveness by modulating the relationship between the Th1/Th2 cytokine imbalance (6).

Liriope platyphylla has also been considered to be an effective therapeutic drug for human subjects suffering from Alzheimer's disease (AD). AD is the most common form of dementia, characterized by the progressive deterioration of cognitive functions, with loss of memory concurrent with pathogenic alterations. In particular, spicatoside A isolated from Liriope platyphylla induced neuritic outgrowth, similar to the effects of nerve growth factor (NGF) in its activation of ERK1/2 and PI3-K/Akt in PC12 cells (11). Furthermore, the butanol fraction of Liriope platyphylla induced the expression and secretion of NGF through a PKC-dependent pathway, increasing the neuritic outgrowth of PC12 cells (10). Furthermore, subchronic administration of ethanolic (70\%) extracts improved learning and memory by enhancement of BDNF or NGF expression (15). Based on these results, we selected Liriope platyphylla in this study as a therapeutic plant having a great potential for new drug development. In addition, the current study provides useful information on the study of the insulin action mechanism, and can contribute to the development of diabetes therapies.

Diabetes is a major worldwide health problem characterized by abnormal insulin regulation, as well as derangement in carbohydrate and lipid metabolism (16). Also, this disease is diagnosed by the presence of hyperglycemia and markedly increases cardiovascular mortality and serious morbidity related to development of nephropathy, retinopathy, and neuropathy (17). Of the two types of diabetes, type 2 or noninsulin-dependent diabetes mellitus (NIDDM) accounts for more than $90 \%$ of the cases, and is characterized by the triad of (a) resistance to insulin action on glucose uptake in peripheral tissue, (b) impaired insulin action that inhibits hepatic glucose production, and (c) dysregulated insulin secretion (18). In this disease, the production and secretion of insulin is very important to the supply and activation of circulating insulin.

Many compounds isolated from medical plants have been widely investigated as insulin regulators. Our study aimed to determine whether LP9M80-H, a novel extract isolated from Liriope platyphylla, would contribute to increasing insulin levels in vitro and in vivo. Ten novel extracts have been isolated from Liriope platyphylla with various chemicals such as ethanol, methanol, and butanol. LP9M80-H extracts having especially high stimulation ability were purified with hexane from the LP-H extract. However, a homoisoflavoneenriched fraction from Liriope platyphylla with $70 \%$ ethanol, and sequentially separated with Diaion HP-20 and silica gel 


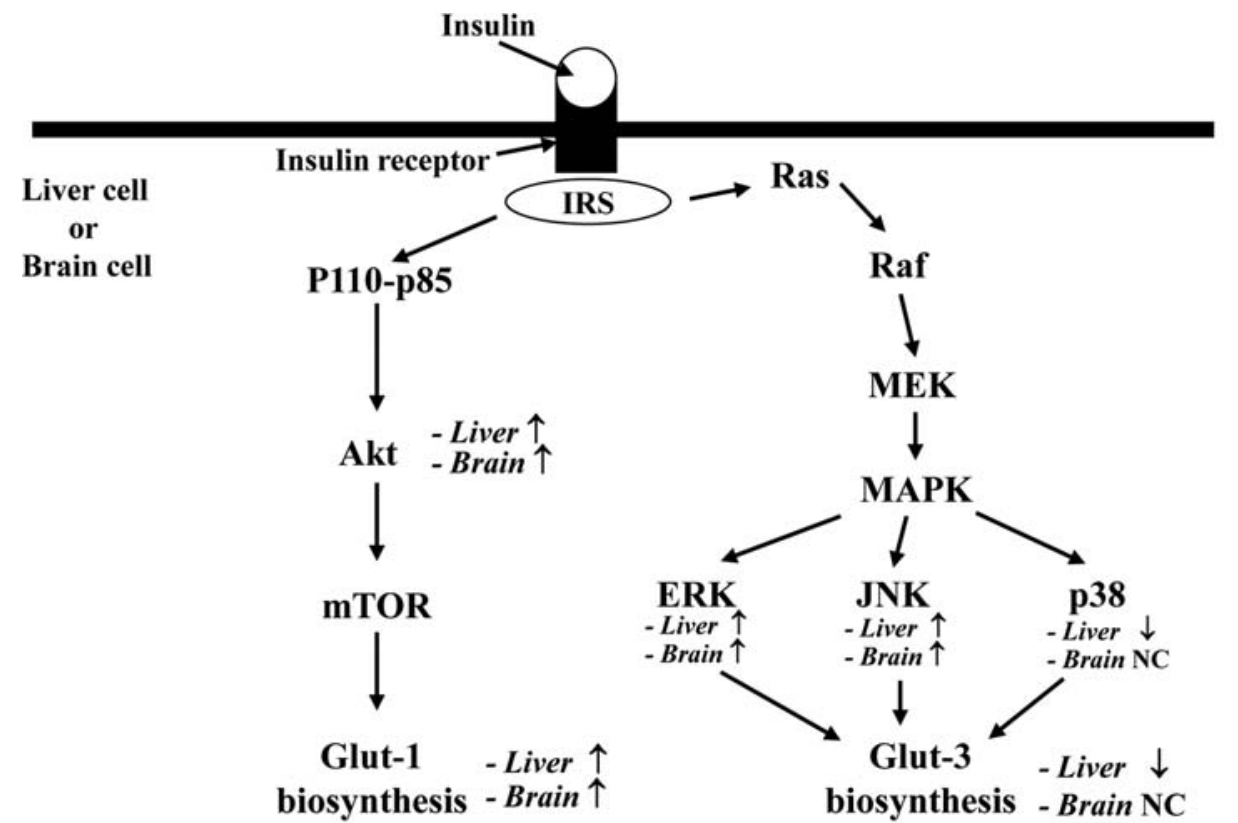

* NC : No change

Figure 10. Regulation pathway of Glut-1 and Glut-3 expression by insulin secreted from pancreatic islets. The regulation starts in the middle with insulin binding to the insulin receptor on the membrane of liver or brain cells. Also, this signal is transferred into two target processes involving Glut-1 and Glut- 3 biosynthesis via two major subpathways. 'NC' in the above diagram indicates 'no change.'

column chromatography, was the first to show the insulin sensitizing effect (9).

Some new drugs have already been developed to treat type 2 diabetes patients. Troglitazone, originally discovered from a Japanese plant, was approved as the first drug to reduce insulin resistance via either enhancing or mimicking insulin action, while it did not affect insulin secretion from ß-pancreatic cells $(19,20)$. However, this drug showed a severe adverse effect on the livers of treated animals. Furthermore, several agents originating from medicinal plants have been reported as insulin sensitizers. One extract of Polygonatum odoratum Druce showed an antihyperglycemic effect by promoting insulin sensitivity (21). However, some active compounds such as homoisoflavonoids, sterols, and steroidal glycosides have been isolated from Liriope platyphylla, but their functions have not been directly studied. Therefore, in this study, ten fractions were screened for their function as insulin stimulators and glucose regulators in a pancreatic $\beta$ cell line. LP9M80-H was selected as a potential candidate for use in diabetes therapy. In animal experiments, LP9M80-H significantly induced an increase in glucose concentration and a decrease in insulin concentration. Immunohistochemical analysis showed that insulin secretion in the pancreas was significantly increased in LP9M80-H-treated mice (Fig. 3). Furthermore, we investigated whether LP9M80-H treatment could affect the insulin signaling pathway in several target tissues. As a first step we selected two main organs, the liver and the brain, that are tightly correlated with insulin action.

One main pathway of the insulin signaling pathway, the MAPK family, is involved in the control of growth and differentiation, as well as in apoptosis signaling (22-24). The MAPK pathway, involving ERK1/2, JNKs, and p38 MAP kinase (p38), has been well characterized in various studies. In particular, several studies showed that the MAPK signal pathways are activated by different stimuli. p38 and JNK are activated in response to many cytotoxic stresses such as hydrogen peroxide $\left(\mathrm{H}_{2} \mathrm{O}_{2}\right)$, UV radiation, tumor necrosis factor (TNF- $\alpha$ ), heat shock, and X-rays (25-27), but ERK is activated by various growth factors and mitogens in processes of cell differentiation, growth, and survival (25).

Generally, the activation pathway of ERK involves three stages of phosphorylation. In these stages, activated Raf (MAPKKK) phosphorylates MEK1/2 (MAPKK), which in turn phosphorylates ERK1/2 (MAPK) (28). Furthermore, ERK mediates glucose metabolism in glomerular podocytes and umbilical vein endothelial cells under high glucose conditions $(29,30)$, but there have been few studies on the mechanism of the correlation between glucose concentration and the MAPK signaling pathway in the liver and the brain. In this study, of members of the MAPK signaling pathway, the highest activation was observed in the ERK pathway in the liver and the brain after LP9M80-H treatment. However, p38 activation was decreased by LP9M80-H in the liver, while it was unchanged in the brain (Figs. 4 and 7). It is well known that basal or constitutive glucose uptake is mediated by Glut-1 and Glut-3 (31). Therefore, we investigated to find the constituent of MAPK that mediates the signal for Glut-3 expression in the liver and brain. Western blot analysis of Glut-3 and MAPK members showed that the phosphorylation level of p38 corresponds to Glut-3 expression in LP9M80-H treatment conditions. Therefore, these results suggest that Glut-3 biosynthesis may be regulated by the p38 pathway of the MAPK family in the liver and the brain after LP9M80-H treatment (Fig. 10). 
The second main pathway mediated by Akt is the cellular homologue of the transforming oncogene v-Akt (32). The primary mechanism of Akt activation involves PI3-K, PDK1, and PDK2. This signaling pathway also leads to a specific endpoint response such as Glut-1 biosynthesis. In this study, Western blot analysis showed that LP9M80-H treatment significantly induced the activation of Akt, regardless of the organ. Furthermore, this activation corresponded with Glut-1 expression (Fig. 10).

Taken together, our results show that LP9M80-H, a novel extract of Liriope platyphylla, could be a potential therapeutic compound for diabetes. Moreover, this study suggests that p38 MAPK and Akt in the insulin signaling pathway tightly associate with Glut biosynthesis after LP9M80-H treatment.

\section{Acknowledgements}

This research was supported by grants to Dr Dae Youn Hwang from the Korea IPET (108160032SB020).

\section{References}

1. Roith DL and Zick Y: Recent advances in our understanding of insulin action and insulin resistance. Diabetes Care 24: 588-597, 2001.

2. Taha $\mathrm{C}$ and Klip A: The insulin signaling pathway. J Membr Biol 169: 1-12, 1999.

3. Feinstein R, Kanety H, Papa MZ, Lunenfeld B and Karasik A: Tumor necrosis factor-alpha suppresses insulin-induced tyrosine phosphorylation of insulin receptor and its substrates. J Biol Chem 268: 26055-26058, 1993.

4. Virkamaki A, Ueki K and Kahn CR: Protein-protein interaction in insulin signaling and the molecular mechanism of insulin resistance. J Clin Invest 103: 931-943, 1999.

5. Czech MP and Corvera S: Signaling mechanisms that regulate glucose transport. J Biol Chem 274: 1865-1868, 1999.

6. Lee YC, Lee JC, Seo YB and Kook YB: Liriopis tuber inhibit OVA-induced airway inflammation and bronchial hyperresponsiveness in murine model of asthma. J Ethnopharmacol 101: 144-152, 2005.

7. Huh MK, Huh HW, Choi JS and BK Lee: Genetic diversity and population structure of Liriope platyphylla (Liliaceae) in Korea. J Life Sci 17: 328-333, 2007.

8. Jeong S, Chae K, Jung YS, Rho YH, Lee J, Ha J, Yoon KH, Kim GC, Oh KS, Shin SS and Yoon M: The Korean traditional medicine Gyeongshingangjeehwan inhibits obesity through the regulation of leptin and PPARalpha action in OLETF rats. J Ethnopharmacol 119: 245-251, 2008.

9. Choi SB, Wha JD and Park S: The insulin sensitizing effect of homoisoflavone-enriched fraction in Liriope platyphylla Wang et Tang via PI3-kinase pathway. Life Sci 75: 2653-2664, 2004.

10. Hur J, Lee P, Kim J, Kim AJ, Kim H and Kim SY: Induction of nerve growth factor by a butanol fraction of Liriope platyphylla in C6 and primary astrocyte cells. Biol Pharm Bull 27: 1257-1260, 2004.

11. Hur J, Lee P, Moon E, Kang I, Kim SH, Oh MS and Kim SY: Neurite outgrowth induced by spicatoside A, a steroidal saponin, via the tyrosine kinase A receptor pathway. Eur J Pharmacol 620: 9-15, 2009.

12. Hwang DY, Chae KR, Shin DW, Hwang JH, Lim CH, Kim YJ, Kim BJ, Goo JS, Shin YY, Jang IS, Cho JS and Kim YK: Xenobiotic response in humanized double transgenic mice expressing tetracycline-controlled transactivator and human CYP1B1. Arch Biochem Biophys 395: 32-40, 2001.
13. Van der Does FE, De Neeling JN, Snoek FJ, Kostense PJ, Grootenhuis PA, Bouter LM and Heine RJ: Symptoms and well-being in relation to glycemic control in type II diabetes. Diabetes Care 19: 204-210, 1996.

14. Kim SW, Chang IM and Oh KB: Inhibition of the bacterial surface protein anchoring transpeptidase sortase by medicinal plants. Biosci Biotechnol Biochem 66: 2751-2754, 2002.

15. Mun JH, Lee SG, Kim DH, Jung JW, Lee SJ, Yoon BH, Shin BY, Kim SH and Ryu JH: Neurotrophic factors mediate enhancing property of ethanolic extract of Liriope platyphylla in mice. J Appl Pharmacol 15: 83-88, 2007.

16. Atalay $M$ and Laaksonen DE: Diabetes, oxidative stress and physical exercise. J Sports Sci Med 1: 1-14, 2002.

17. Zimmet PZ, McCarty DJ and de Courten MP: The global epidemiology of non-insulin-dependent diabetes mellitus and the metabolic syndrome. J Diabetes Complications 11: 60-68, 1997.

18. Kahn BB: Type 2 diabetes: when insulin secretion fails to compensate for insulin resistance. Cell 92: 593-596, 1998.

19. Iwanishi M and Kobayash M: Effects of pioglitazone on insulin receptors skeletal muscle from high-fat-fed rats. Metabolism 42: 1017-1021, 1993.

20. Kreagen EW, James DE and Jenkins AB: A potent in vivo effect of ciglitazone on muscle insulin resistance induced by high fact feeding of rats. Metabolism 38: 1089-1093, 1989.

21. Choi SB and Park S: A steroidal glycoside from Polygonatum (Mill.) odoratum Druce improves insulin resistance but does not alter insulin secretion in $90 \%$ pancreatectomized rats. Biosci Biotech Biochem 66: 2036-2043, 2002.

22. Marshall CJ: MAP kinase kinase kinase, MAP kinase kinase and MAP kinase. Curr Opin Genet Dev 4: 82-89, 1994.

23. Waskiewicz AJ and Cooper JA: Mitogen and stress response pathways: MAP kinase cascades and phosphatase regulation in mammals and yeast. Curr Opin Cell Biol 7: 798-805, 1995.

24. Fanger GR, Gerwins P, Widmann C, Jarpe MB and Johnson GL: MEKKs, GCKs, MLKs, PAKs, TAKs, and tpls: upstream regulators of the c-Jun amino-terminal kinases? Curr Opin Genet Dev 7: 67-74, 1997.

25. Huot J, Houle F, Marceau F and Landry J: Oxidative stressinduced actin reorganization mediated by the p38 mitogenactivated protein kinase/heat shock protein 27 pathway in vascular endothelial cells. Circ Res 80: 383-392, 1997.

26. Van Rij AM, Thomson CD, McKenzie JM and Robinson MF: Selenium deficiency in total parenteral nutrition. Am J Clin Nutr 32: 2085-2086, 1979.

27. Scorziello A, Santillo M, Adornetto A, Dell'Aversano C, Sirabella R, Damiano S, Canzoniero LMT, Di Renzo GF and Annunziato L: NO-induced neuroprotection in ischemic preconditioning stimulates mitochondrial Mn-SOD activity and expression via RAS/ERK1/2 pathway. J Neurochem 103: 1472-1480, 2007.

28. Pearson G, Robinson F, Beers Gibson T, Xu BE, Karandikar M, Berman K and Cobb MH: Mitogen-activated protein (MAP) kinase pathways: regulation and physiological functions. Endocr Rev 22: 153-183, 2001.

29. Hoshi S, Nomoto K, Kuromitsu J, Tomari S and Nagata M: High glucose induced VEGF expression via PKC and ERK in glomerular podocytes. Biochem Biophys Res Commun 290: 177-184, 2002.

30. Xin X, Khan ZA, Chen S and Chakrabarti S: Extracellular signal-regulated kinase (ERK) in glucose-induced and endothelin-mediated fibronectin synthesis. Lab Invest 84: 14511459,2004

31. Bell G, Kayano T, Buse J, Burant C, Takeda J, Lin D, Fukumoto $\mathrm{H}$ and Seino S: Molecular biology of mammalian glucose transporters. Diabetes Care 13: 198-208, 1990.

32. Bellacosa A, Testa JR, Staal SP and Tsichlis PN: A retroviral oncogene, akt, encoding a serine-threonine kinase containing an SH2-like region. Science 254: 274-277, 1991. 